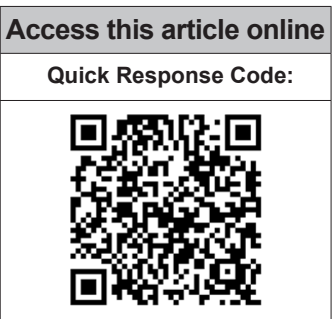

Website:

www.jponline.org

DOI:

10.4103/JLP.JLP_157_17
Department of Pathology, IPGME and $R,{ }^{2}$ Department of Paediatric Surgery, Apollo Gleneagles Hospital, ${ }^{1}$ Department of Surgery,

Calcutta National Medical College and Hospital, Kolkata, West Bengal, India

Address for correspondence: Dr. Chhanda Das, Department of Pathology, IPGMER, Kolkata,

West Bengal, India. E-mail: chhhdas@gmail.

Submission: 16-10-2017 Accepted: 30-05-2018

\title{
Evaluation of diagnostic utility of imprint cytology in paediatric renal tumours with special references to $\mathrm{Ki}$ 67 proliferative marker
}

\author{
Namrata Maity, Chhanda Das, Madhumita Mukhopadhyay, Tamanna Parvin, \\ Ashis Kumar Saha1, Biswanath Mukhopadhyay²
}

\begin{abstract}
:
BACKGROUND: Pediatric renal neoplasms comprise about $7 \%-8 \%$ of all neoplasms in children. Wilms tumour (WT) is the most common among pediatric renal tumours.

AIMS AND OBJECTIVES: The study was undertaken to study the epidemiological occurrence of pediatric renal tumours in a tertiary care hospital and to ascertain the validity and reliability of touch smear imprint cytology in intraoperative diagnosis of renal tumours and correlate with subsequent histopathological diagnosis and to assess the expression of proliferation marker Ki-67 in different components and stages of WT.
\end{abstract}

MATERIALS AND METHODS: It was a single-institution-based prospective and observational study, conducted for 2 years (from October 2013 to September 2015) in the department of pathology at our hospital. A total of fifty cases were enrolled in this study, all were below 15 years of age.

RESULTS: Imprint cytology showed sensitivity, specificity, and diagnostic accuracy of $83 \%, 98 \%$, and $95.74 \%$, respectively, in diagnosing benign and malignant renal tumours. There was statistically significant correlation of imprint cytology with confirmatory histopathological examination of excision specimen $(P<0.001)$. Immunohistochemical analysis of Ki-67 was done in all WT cases. Epithelial component had higher proliferative index than blastemal component with $P=0.0082$, which was highly statistically significant.

CONCLUSION: Imprint cytology is found to be a less expensive, simple, and rapid method, which can be used as an adjunct to histopathology. Correlation between proliferation index as measured with $\mathrm{Ki}-67$ antibody and tumour stage was found. $\mathrm{Ki}-67$ is thus a relevant marker for assessing the proliferative activity.

Key words:

Imprint cytology, kidney tumour, proliferative index

\section{Introduction}

Dediatric renal tumours comprise $7 \%-8 \%$ of all tumours in children, in which diagnosis and treatment are highly dependent on the histopathological findings. ${ }^{[1,2]}$ The diagnosis of a renal neoplasm in children is suggested by clinical history,

This is an open access journal, and articles are distributed under the terms of the Creative Commons Attribution-NonCommercial-ShareAlike 4.0 License, which allows others to remix, tweak, and build upon the work non-commercially, as long as appropriate credit is given and the new creations are licensed under the identical terms.

For reprints contact: reprints@medknow.com age at presentation, and distinctive imaging features. In a neonate, most renal masses are due to developmental abnormalities such as congenital hydronephrosis or multicystic dysplastic kidney. Beyond the $1^{\text {st }}$ year of life and during the first decade, primary tumours of the kidney are more common. ${ }^{[3]}$ Wilms tumour (WT) is the most common $(85 \%)$, followed by mesoblastic nephroma $(5 \%)$, clear-cell sarcoma of the

How to cite this article: Maity $\mathrm{N}$, Das C, Mukhopadhyay M, Parvin T, Saha AK, Mukhopadhyay B. Evaluation of diagnostic utility of imprint cytology in paediatric renal tumours with special references to Ki 67 proliferative marker. J Lab Physicians 2018;10:437-42. 
kidney $(4 \%)$, rhabdoid tumour of the kidney $(2 \%)$, and miscellaneous rare tumours (4\%). Their treatment and prognosis are very different and depend on accurate histology and their stage. ${ }^{[1]}$ Most renal tumours present with nonspecific features of an abdominal mass, usually detected incidentally by parents. Abdominal pain, hematuria, and hypertension may also occur. Most kidney tumours present with nonspecific radiological findings. A dramatic improvement in overall survival rates has resulted from the coordinated use of modern surgical technique and anesthesia, multiple drug chemotherapy, and radiation therapy. ${ }^{[4]}$ Large cooperative cancer groups, especially the National Wilms Tumour Study and the International Society of Paediatric Oncology (SIOP), have laid down guidelines for standardized treatment of this tumour and thus achieved a 5-year survival rate of more than $90 \% .^{[5]}$ The SIOP protocols advocate preoperative therapy followed by surgical removal. This approach allows for tumour shrinkage by therapy prior to resection.

Fine-needle aspiration cytology (FNAC), image-guided aspiration cytology, and imprint cytology are the common techniques practiced routinely to study the cytology of tissues. ${ }^{[6]}$

FNAC as a preoperative investigation has been discouraged because deep location makes it relatively inaccessible for aspiration without image guidance and has the risk of needle tract seeding and dissemination. ${ }^{[7]}$ Intraoperative imprint cytology provides rapid diagnosis (within $20 \mathrm{~min}$ ) without the fear of dissemination and helps the surgeons to plan the extent of surgery while the patient is still in operation theater after a proper diagnosis has been made. Identification of factors predictive of the aggressive growth of WT helps in recognition of the biologic course of cancer prognosis besides enabling stratification of patients for optimal treatment strategy. ${ }^{[8]}$ The Ki-67 monoclonal antibody is used for this purpose. MIB-1 recognizes the Ki-67 nuclear antigen, which is associated with cell proliferation and is found throughout the cell cycle. ${ }^{[9]}$

This study was carried out to correlate the result of touch smear imprint cytology in intraoperative diagnosis of renal tumours with subsequent histopathological diagnosis and assess the expression of proliferation marker Ki-67 in different stages of WT.

\section{Materials and Methods}

The study was performed after obtaining approval from the ethical committee. Children below 15 years of age, attending the pediatric surgery outdoor of IPGME and R, diagnosed clinically and radiologically as having kidney tumour and subsequently undergoing resection were selected for the study. The specimens were sent to the Department of Pathology for further examination. Imprint smears were taken from the fresh specimens and compared to the histopathological diagnosis. Children who received preoperative chemotherapy were also included in this study. Children with inoperable tumours and those with completely necrotic tumours after preoperative chemotherapy and very seriously patients were excluded from the study. May-GrunwaldGiemsa and Papanicolaou staining were performed on touch imprint smears. Hematoxylin and eosin staining was done on tissue sections obtained from subsequent excision surgeries to see the histological features.

Following histopathological diagnosis, immunohistochemical staining was done by Ki-67 antibody of histological sections. All identifiable nuclear staining in cells was recorded as positive regardless of intensity. At least 1000 cells were observed under a microscope (objective lens $\times 40$ ) and counted vertically from one corner to another. The percentage of stained cells was evaluated three times independently by two examiners. The result of Ki-67 immunostaining was interpreted as labeling index $=$ number of nuclei showing positive staining (brown color)/total number of nuclei $(1000) \times 100 \%$. Tonsil was used as a positive control of Ki-67 antibody and negative control was achieved by omitting the primary antibody. The cutoff value of $5 \%$ was statistically known to be the lowest value at which discrimination with a significant probability value was achieved. In full sections, at least three high-power $(\times 400)$ fields were selected to represent the spectrum of staining seen on an initial overview of the whole section. ${ }^{[10]}$

Results obtained from imprint smears were correlated with histopathological reports.

\section{Statistical analysis}

All data were copied into Microsoft Excel version 12.0 and contingency tables were prepared for qualitative data. Data were analyzed with the help of GraphPad Instat (Graph Pad Software, San Diego, CA, USA) version 3, Prism GraphPad version 5 (GraphPad Software Inc, California Corporation), and MedCalc version 11.6 (MedCalc Software 2011, Mariakerke, Belgium). Chi-square test for independence was done for association of Ki-67 with WT. All the categorical data were compared by Fisher's exact test. $P<0.05$ was considered statistically significant.

\section{Results}

Among the total fifty cases, 33 cases (66\%) belonged to the category of nephroblastoma/WT followed by clear-cell sarcoma (12\%), multicystic renal dysplasia $(10 \%)$, mesoblastic nephroma $(6 \%)$, rhabdoid tumour $(4 \%)$, 
and mature teratoma constituting $2 \%$ of all cases. Most cases occurred in children between 1 and 3 years of age. Imprint smears diagnosed a total of 38 cases as malignant, 3 cases as suspicious of malignancy, 6 as benign, and 3 as unsatisfactory. On histopathological examination (HPE), 44 cases were found to be of malignant category with only 6 benign cases. Hence, a comparison between imprint and histopathological diagnosis was done. Among the fifty children included in the study, five cases were diagnosed as benign from imprint cytology, all of which were later confirmed as benign from subsequent final HPE (true negative $=5$ ). Only a single case was found to be benign on HPE, which had previously been diagnosed as malignant on imprint cytology (false positive $=1$ ). Forty cases were diagnosed as malignant from imprint cytology, and this corroborated with their histopathological diagnosis as well (true positive $=40$ ). One case which was thought to be benign in imprint cytology was later on diagnosed as malignant from histopathology (false negative $=1$ ). Three cases which had been found unsatisfactory on imprint cytology, with very scanty cells, could not be used for a provisional diagnosis. Hence, these three cases were excluded during this calculation.

On statistical analysis comparing the imprint cytology with histopathological diagnosis of renal neoplasms [Figures 1-4], $P<0.001$ was obtained, which was considered highly statistically significant. Overall sensitivity, specificity, and diagnostic accuracy

Table 1: Distribution of cases in benign and malignant categories, as per their diagnoses from imprint and histopathological examination

\begin{tabular}{lcccc}
\hline \multirow{2}{*}{$\begin{array}{l}\text { Imprint } \\
\text { cytology }\end{array}$} & \multicolumn{2}{c}{ Histopathological category } & Total & $P$ \\
\cline { 2 - 3 } Benign & Benign & Malignant & & \\
Malignant & $5(\mathrm{TN})$ & $1(\mathrm{FN})$ & 6 & $<0.001$ \\
Total & $1(\mathrm{FP})$ & $40(\mathrm{TP})$ & 41 & \\
\hline
\end{tabular}

$\mathrm{TN}=$ True negative, $\mathrm{FN}=$ False negative, $\mathrm{FP}=$ False positive, $\mathrm{TP}=$ True positive

Table 2: Overall Efficacy of Imprint Cytology In Diagnosing Paediatric Renal Tumours

\begin{tabular}{lc}
\hline Sensitivity & 83 \\
Specificity & 98 \\
PPV & 83 \\
NPV & 98 \\
False-positive rate $(\alpha)$ & 2 \\
False-negative rate $(\beta)$ & 17 \\
Positive likelihood ratio & 34.17 \\
Negative likelihood ratio & 0.17 \\
Matthews correlation coefficient & 0.808943089 \\
$t$-test & 0.282533409 \\
Kohen's Kappa $(\kappa)$ & 0.808943089 \\
Chi-square test $\left(\chi^{2}\right)$ & 30.75627933 \\
Strong agreement & $P<0.001$
\end{tabular}

$\mathrm{PPV}=$ Positive predictive value, NPV=Negative predictive value of imprint cytology were $83 \%, 98 \%$, and $95.74 \%$, respectively [Tables 1 and 2]. Staging according to the SIOP WT 2001 showed that most of the cases (42\%)

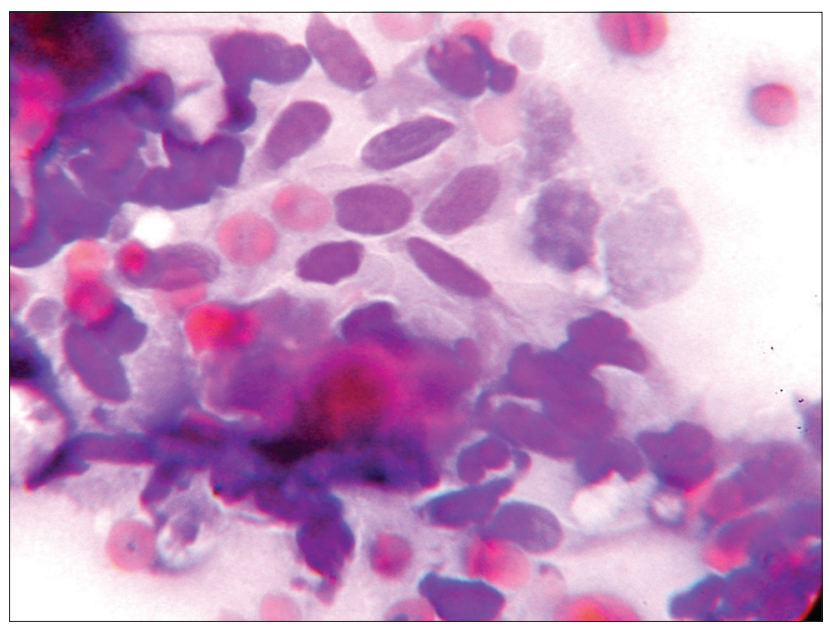

Figure 1: Photomicrograph showing imprint cytology of congenital mesoblastic nephroma $(\times 400)$

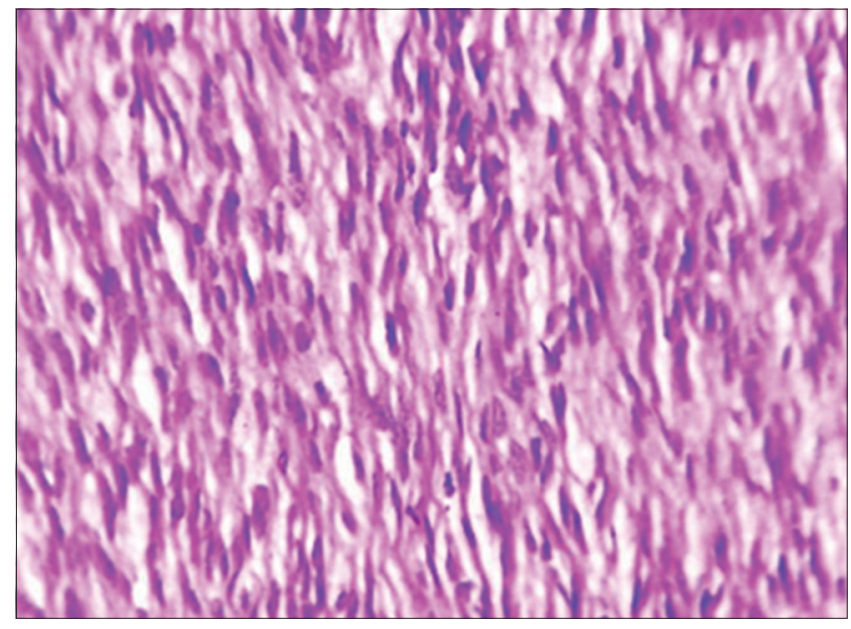

Figure 2: Photomicrograph showing histology of congenital mesoblastic nephroma $(\times 100)$

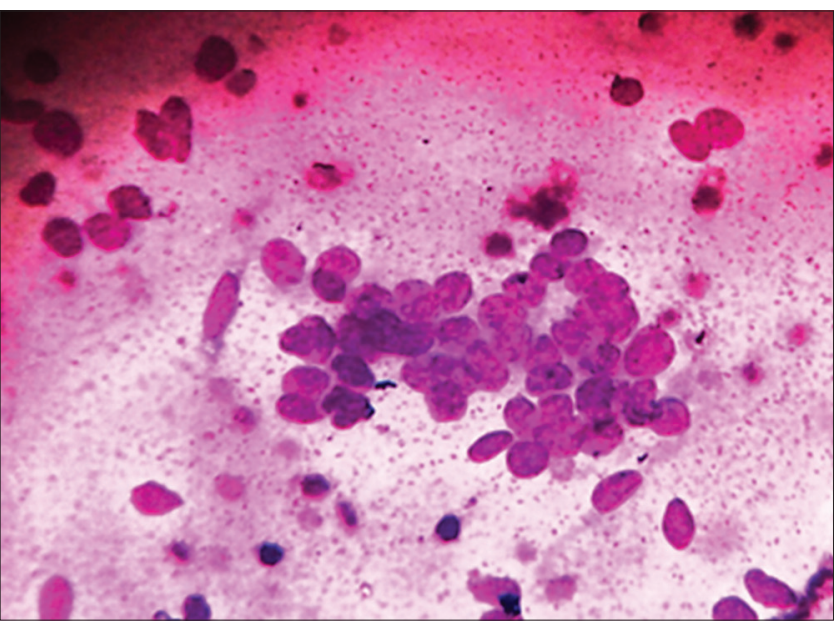

Figure 3: Imprint cytology of blastemal cells in Wilms tumour $(\times 400)$ 
belonged to Stage III, with $30 \%$ cases being of Stage I, $24 \%$ cases of Stage II, and $4 \%$ of Stage IV [Table 3]. Fourteen out of $33 \%$ cases of WT showed triphasic components (blastemal, epithelial, and stromal), with blastemal-type WT being the second-most common (11 cases) [Table 4]. Ki-67 labeling index was determined for blastemal and epithelial components separately [Figures 5 and 6]. Ki-67 labeling index was determined for blastemal and epithelial components separately. Of the 33 cases of WT, the range of $\mathrm{Ki}-67-$ positive cells in the epithelial component in Stage I was $18 \%-66 \%$ (mean $45 \%$ ) and in blastemal this was $10 \%-50 \%$ (mean 38\%); in Stage II, the epithelial component was $60 \%-88 \%$ (mean $74 \%$ ) and in blastemal it was 38\%-56\% (mean $48 \%$ ); and in Stage III, in the epithelial component, this was $58 \%-98 \%$ (mean $78 \%$ ) and in blastemal it was $48 \%-88 \%$ (mean $68 \%$ ). The single case of Stage IV showed very low proliferative index $(<5 \%)$ and hence was considered to be negative. On analyzing the data, epithelial component especially showed higher proliferative index along with increasing tumour stage compared with blastemal component with $P=0.0082$, which is highly statistically significant [Table 5].

Table 3: Distribution of Wilms tumour according to tumour stage

\begin{tabular}{lc}
\hline Tumour stage & Number of cases \\
\hline Stage I & 10 \\
Stage II & 8 \\
Stage III & 14 \\
Stage IV & 1 \\
Stage V & 0 \\
\hline
\end{tabular}

Table 4: Distribution of various histopathological variants of Wilms tumour (International Society of Pediatric Oncology)

\begin{tabular}{llc}
\hline Risk category & Histopathological subtype & Number of cases \\
\hline Low risk & CPDN & 1 \\
Intermediate & Epithelial type & 2 \\
risk & Triphasic/mixed type & 14 \\
& Regressive type & 1 \\
& Rhabdomyoblastic differentiation & 1 \\
High risk & Diffuse anaplasia & 3 \\
& Blastemal type & 11 \\
\hline
\end{tabular}

$\overline{\mathrm{CPDN}}=$ Cystic partially differentiated nephroblastoma

Table 5: Distribution of the Ki-67 expression in the blastemal and epithelial components of the Wilms tumour tissue along with advancing tumour stage

\begin{tabular}{|c|c|c|c|c|c|c|}
\hline \multirow[t]{2}{*}{ Stage } & \multicolumn{2}{|c|}{ Blastemal (\%) } & \multicolumn{2}{|c|}{ Epithelial (\%) } & \multirow{2}{*}{$\begin{array}{c}\text { Overall } \\
(\%)\end{array}$} & \multirow[t]{2}{*}{$P$} \\
\hline & Range & Mean & Range & Mean & & \\
\hline Stage I & $10-50$ & 38 & $18-66$ & 45 & 41.5 & 0.0082 \\
\hline Stage II & $38-56$ & 48 & $60-88$ & 74 & 61 & \\
\hline Stage III & $48-88$ & 68 & $58-98$ & 78 & 73 & \\
\hline Stage IV & 0 & 0 & 0 & 0 & 0 & \\
\hline
\end{tabular}

\section{Discussion}

In this study, all children were below 12 years of age, with the youngest one being 9 days old and 11 years

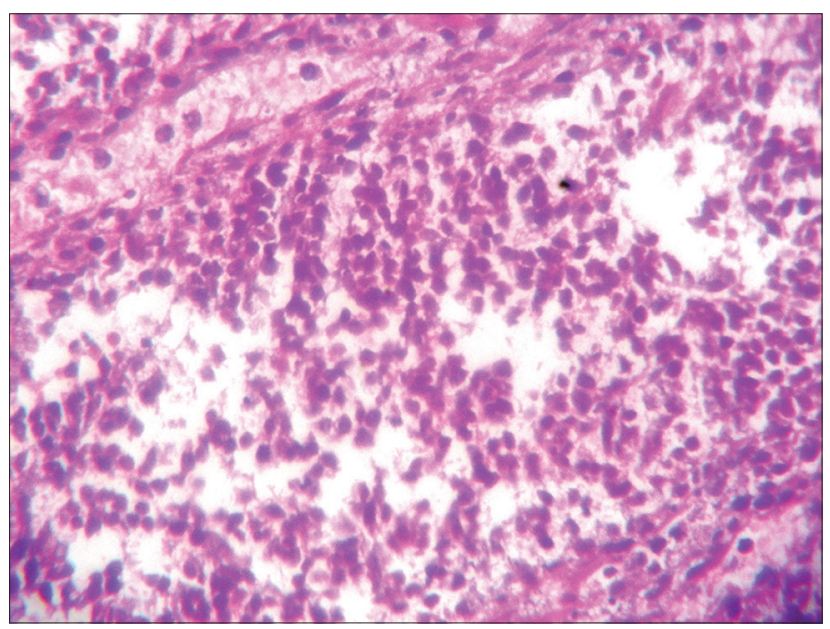

Figure 4: Histology of blastemal cells ( $\times 400)$

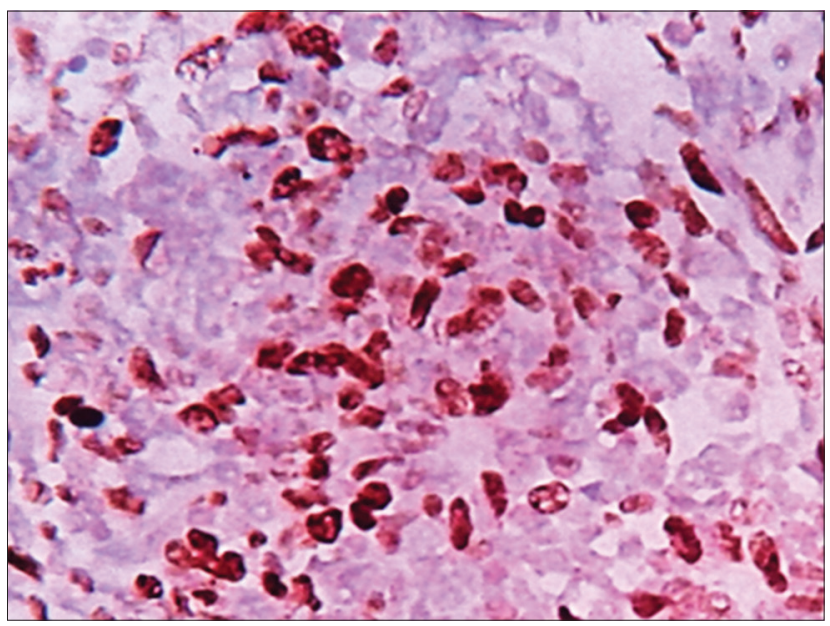

Figure 5: Ki67-positive cells in blastemal cells $(\times 400)$

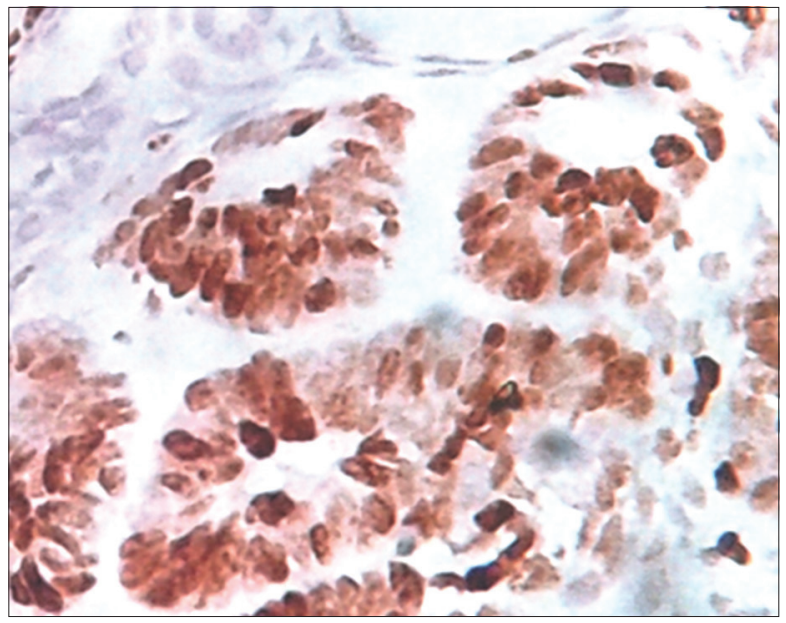

Figure 6: Ki67-positive cells in epithelial cells ( $\times 400)$ 
being the oldest one in this study. The mean age was 2.9 years. Vujanić et al. showed in their study that $7 \%-8 \%$ of renal tumours in children occur in the first 15 years of life. ${ }^{[11]}$ The mean age of WT cases was 3.2 years for males and 2.8 years for females. The median age at diagnosis, as noted by Breslow et al., was 3.5 years for males with unilateral tumours and 3.9 years for females with unilateral tumours. ${ }^{[12]}$ In our study, females were found to be slightly more affected than males accounting for $54 \%$ of the total cases, whereas males accounted for $46 \%$ of cases. Breslow et al. had also reported a similar female preponderance in their study. ${ }^{[13]}$ Most cases (54\%) were seen involving the right kidney in our study, similar to that demonstrated by Blute et al. in their study. They noted that $4.4 \%$ of WT cases were bilateral ${ }_{,}^{[14]}$ but in our study, we did not come across any case of bilateral WT. ${ }^{[14]}$

The most common clinical feature with which the child presented was usually a nonspecific abdominal mass similar to that reported by Sebire and Vujanic. ${ }^{[1]}$ They had also seen a few cases having features of abdominal pain, hematuria, and hypertension. In our study, the two cases of rhabdoid tumour presented with hematuria, but others presented with abdominal mass only.

In our study, nephroblastoma or WT was found to be the most common accounting for $66 \%$ of cases followed by clear-cell sarcoma (12\%), multicystic renal dysplasia (10\%), mesoblastic nephroma (6\%), rhabdoid tumour (4\%), and mature teratoma accounting for $2 \%$ of cases. Sebire and Vujanic also noted WT to be the most common (85\%), followed by mesoblastic nephroma (5\%), clear-cell sarcoma of the kidney $(4 \%)$, rhabdoid tumour of the kidney ( $2 \%)$, and miscellaneous rare tumours (4\%). ${ }^{[1]}$

Most of the renal tumours (42 out of 50) were found to be solid on gross examination, with a total of $84 \%$ cases, whereas partially cystic tumours and completely cystic ones constituted 8\% each. Among the cases of nephroblastoma, 32 of the total 33 cases were solid except for a single case of cystic partially differentiated nephroblastoma, which was predominantly cystic in nature. Charles et al. also found majority of the WT cases to be solid on gross examination. ${ }^{[15]}$

We have done intraoperative imprint cytology in all the cases and correlated with the histopathological diagnosis done later. On analyzing the results of all the fifty cases, imprint cytology showed a sensitivity of $83 \%$ and specificity of $98 \%$, with a diagnostic accuracy of $95.74 \%$. Shet and Viswanathan in their study on Indian population also concluded that pediatric renal tumours present a unique spectrum very much amenable to an accurate cytological diagnosis with a little bit of experience. ${ }^{[16]}$
Staging according to the SIOP WT 2001 Staging Criteria was done for all the 33 cases of WT, since all of them had undergone preoperative chemotherapy. It was found that most of the cases $(42 \%)$ belonged to Stage 3, with $30 \%$ cases being of Stage 1, 24\% cases of Stage 2, and $4 \%$ of Stage 4 . Gupta et al. in their study comprising of 202 cases over a period of 17 years had reported $19.3 \%$ as Stage I, $15.8 \%$ as Stage II, $43.0 \%$ as Stage III, $15.3 \%$ as Stage IV, and $6.4 \%$ as Stage V. ${ }^{[5]}$

Vujanic et al. in their study had stated that a major disadvantage of preoperative chemotherapy was the fact that it significantly alters the histological features of WT, resulting in different histological patterns and distribution of subtypes from those treated with immediate surgery. Preoperative chemotherapy is more likely to destroy blastema and (less differentiated) epithelial elements, while it induces maturation especially in the stromal component, where rhabdomyoblastic differentiation is much more common than in primarily operated tumours. The typical chemotherapy-induced changes of treated WTs are a mixture of coagulative-type necrosis of small round cells or neoplastic tubules consisting of pink, necrotic nuclei, consistent with coagulative necrosis of blastemal cells or neoplastic tubules, fibrosis, hypocellular stroma containing foamy and/or hemosiderin-laden macrophages, and hemorrhage. ${ }^{[17]}$

Since all the 33 cases of nephroblastomas included in this study had received preoperative chemotherapy, they were further categorized into low, intermediate, and high-risk types, based on SIOP Working Classification of Renal Tumour of Childhood (2001) and postchemotherapy changes. Blastemal and epithelial elements were found to be the most predominant histological components in all cases. Majority of the cases belonged to intermediate-risk group, consisting of 14 cases of triphasic/mixed type, two cases of epithelial type, and one case each of regressive type and WT with rhabdomyoblastic differentiation. The high-risk category included 11 cases of blastemal-type WT and three cases showing diffuse anaplasia. Besides the histological components, associated foci of nephrogenic rests were found in 9 out of 33 cases (27\%). Charles et al. had shown in their study that microscopic rests are associated with $30 \%-40 \%$ of WT cases and are believed to be precursors of WT. ${ }^{[15]}$ In our study, we investigated the expression of proliferation marker $\mathrm{Ki}-67$ in nephroblastoma and correlated its expression in epithelial and blastemal components in different stages.

We found that the epithelial component had a significantly higher proliferative index compared with the blastemal component, in all stages, with $P=0.0082$, which is highly statistically significant. Epithelial component especially showed higher proliferative index along with increasing tumour stage. Studies by various authors like Ghanem 
et al. also demonstrated similar findings. ${ }^{[10]}$ Juric et al. in their study showed that blastemal component of WT is less differentiated or immature, and hence more sensitive to preoperative chemotherapy. Hence, in cases undergoing chemotherapy, in the histologically preserved tumour tissue, there is reduction of $\mathrm{Ki}-67$ proliferation index along with reduction of blastemal component. The epithelial component is better differentiated and is therefore less sensitive to preoperative chemotherapy ${ }^{[18]}$

\section{Conclusion}

Our study deals with the spectrum of pediatric renal neoplasms frequently seen in our setup. Imprint cytology is found to be a less expensive, simple, and rapid method, which can be used as an adjunct to histopathology. Correlation between proliferation index as measured with Ki-67 antibody and tumour stage was found, according to treatment response within the tumour tissue. Ki-67 is thus a relevant marker for assessing the proliferative activity and tumour cell dynamics of WT.

\section{Limitations}

Since our study is single-institute based and the study period is very short, the number of cases are small and it is not possible to give a generalized result to comment on the whole population. The present study may be reviewed as a component of a large multicentric study to reach a definite conclusion. Survival analysis could not be done as follow-up was not possible for the short time limit. Ki-67 may not be a good clinical prognostic marker in those patients receiving preoperative chemotherapy because epithelial component was better differentiated and therefore less sensitive to preoperative chemotherapy. A larger sample size and further analysis would be required to make any significant comment about the prognostic value of Ki-67 in WT.

\section{Financial support and sponsorship Nil.}

\section{Conflicts of interest}

There are no conflicts of interest.

\section{References}

1. Sebire NJ, Vujanic GM. Paediatric renal tumours: Recent developments, new entities and pathological features.
Histopathology 2009;54:516-28.

2. Murphy WM, Grignon DJ, Perlman EJ. Kidney tumours in children. In: AFIP Atlas of Tumour Pathology $4^{\text {th }}$ Series Fascicle 1: Tumours of the Kidney, Bladder and Related Urinary Structures. Washington DC: American Registry of Pathology; 2004.

3. van den Heuvel-Eibrink MM, Grundy P, Graf N, Pritchard-Jones K, Bergeron C, Patte C, et al. Characteristics and survival of 750 children diagnosed with a renal tumour in the first seven months of life: A collaborative study by the SIOP/GPOH/SFOP, NWTSG, and UKCCSG Wilms tumour study groups. Pediatr Blood Cancer 2008;50:1130-4.

4. Gupta DK. Progress in treatment of Wilms' tumour. In: Hemal AK, editor. Advances in Uro-Oncology. New Delhi: Saurabh Publishers; 1995.

5. Gupta DK, Sharma S, Agarwala S, Carachi R. Saga of Wilms' tumour-Lessons learnt from the past. (Department of Pediatric Surgery, All India Institute of Medical Sciences, New Delhi and *York Hill Children's Hospital, Glasgow, U.K.). J Indian Assoc Pediatr Surg 2005;10:217-28.

6. Suen KC, Wood WS, Syed AA, Quenville NF, Clement PB. Role of imprint cytology in intraoperative diagnosis: Value and limitations. J Clin Pathol 1978;31:328-37.

7. Hajdu SI, Melamed MR. Limitations of aspiration cytology in the diagnosis of primary neoplasms. Acta Cytol 1984;28:337-45.

8. Wen JG, van Steenbrugge GJ, Egeler RM, Nijman RM. Progress of fundamental research in Wilms' tumour. Urol Res 1997;25:223-30.

9. Cattoretti G, Becker MH, Key G, Duchrow M, Schlüter C, Galle J, et al. Monoclonal antibodies against recombinant parts of the Ki-67 antigen (MIB 1 and MIB 3) detect proliferating cells in microwave-processed formalin-fixed paraffin sections. J Pathol 1992;168:357-63.

10. Ghanem MA, Van der Kwast TH, Sudaryo MK, Mathoera RB, van den Heuvel MM, Al-Doray AA, et al. MIB-1 (KI-67) proliferation index and cyclin-dependent kinase inhibitor p27(Kip1) protein expression in nephroblastoma. Clin Cancer Res 2004;10:591-7.

11. Vujanić GM, Sandstedt B, Harms D, Kelsey A, Leuschner I, de Kraker J, et al. Revised International Society of Paediatric Oncology (SIOP) working classification of renal tumours of childhood. Med Pediatr Oncol 2002;38:79-82.

12. Breslow N, Olshan A, Beckwith JB, Green DM. Epidemiology of Wilms tumour. Med Pediatr Oncol 1993;21:172-81.

13. Breslow N, Beckwith JB, Ciol M, Sharples K. Age distribution of Wilms' tumour: Report from the national Wilms' tumour study. Cancer Res 1988;48:1653-7.

14. BluteML,KelalisPP, Offord KP, Breslow N, BeckwithJB, D' AngioGJ, et al. Bilateral Wilms tumour. J Urol 1987;138:968-73.

15. Charles AK, Vujanić GM, Berry PJ. Renal tumours of childhood. Histopathology 1998;32:293-309.

16. Shet $\mathrm{T}$, Viswanathan $\mathrm{S}$. The cytological diagnosis of paediatric renal tumours. J Clin Pathol 2009;62:961-9.

17. Vujanić GM, Sandstedt B. The pathology of Wilms' tumour (nephroblastoma): The international society of paediatric oncology approach. J Clin Pathol 2010;63:102-9.

18. Jurić I, Pogorelić Z, Kuzmić-Prusac I, Biocić M, Jakovljević G, Stepan J, et al. Expression and prognostic value of the Ki-67 in Wilms' tumour: Experience with 48 cases. Pediatr Surg Int 2010;26:487-93. 